\title{
Sesión de cine en Educación Secundaria
}

\section{Francisco Ignacio MORETA VELAYOS, Inés DE LA FUENTE HERMOSÍN, Lucia CARBONELL MUÑOZ, Carolina MORETA MONTERO, Nieves MONTERO SÁNCHEZ, Silvia CONDE BLANCO}

Centro de Salud Parque Coímbra. Gerencia de Atención Primaria. Servicio Madrileño de Salud. Móstoles. Madrid (España).

Autor para correspondencia: Francisco Ignacio Moreta Velayos. Correo electrónico: fimoreta@yahoo.es

Recibido el 3 de junio de 2018; aceptado el 4 de julio de 2018.

Cómo citar este artículo: Moreta Velayos Fl, de la Fuente Hermosín I, Carbonell Muñoz L, Moreta Montero C, Montero Sánchez N, Conde Blanco S. Sesión de cine en Educación Secundaria. Rev Med Cine [Internet] 2019;15(1): 3-7.

DOI: https://dx.doi.org/10.14201/rmc.20246

\section{Resumen}

Tras la experiencia inicial de utilizar el cine como estrategia para la formación continuada en nuestro Centro de Salud, valoramos la posibilidad de compartir la experiencia con el Instituto de Educación Secundaria de nuestra Zona Básica como recurso pedagógico con el objetivo de contribuir a la Educación para la Salud de los alumnos.

En torno a la proyección de las películas seleccionadas se fomenta el debate grupal sobre tópicos y valores saludables de especial interés en la adolescencia y juventud.

De forma breve se describe el desarrollo del proyecto y la acogida del mismo por los alumnos y profesorado que han valorado el cine como una buena herramienta para mejorar la calidad de la educación para la salud en el Instituto.

Palabras clave: educación en salud, educación secundaria, cine, valores sociales.

\section{Movie session in high school}

\section{Summary}

After the initial experience of using cinema as a tool to follow-up training in our Health Center, we assessed the possibility of sharing the experience with the High School of our basic zone as a pedagogical resource with the purpose of improving the students' health education.

Around the selected films proyection, group discussion was promoted on topics and healthy values of special interest in adolescence and youth.

Briefly, the development of the project is described and also the reception of the project by students and teachers who have valued cinema as a good tool to improve the quality of health education in the secondary education level.

Keywords: Health education, Secondary education, Cinema, Social values.

Los autores declaran que el artículo es original y que no ha sido publicado previamente. 


\section{Proyecto de Educación para la Salud en el Instituto}

Durante los dos últimos años y como estrategia de autoformación en nuestro Centro de Salud, "Parque Coímbra" en Mostoles (Madrid), hemos desarrollado sendos ciclos de cine que hemos Ilamado Sesión de cine en la Primaria ${ }^{1}$.

En ellos, tras el visionado de distintas películas, se discutía su utilidad en distintos aspectos para nuestro trabajo, habiéndose destacado en numerosas ocasiones la posibilidad de la aplicación de distintos títulos, en la Educación para la Salud y Humanización de la Medicina, en su relación con la adolescencia y la juventud.

A partir de aquí, surge la propuesta de compartir nuestra experiencia con el Instituto de nuestra zona, I.E.S Gabriel Cisneros, ya que como es sabido, a través de la Educación para la Salud en el medio escolar es posible inculcar conocimientos, actitudes y hábitos positivos de salud en la adolescencia, una etapa en la que la personalidad se está aún forjando y donde ver modelos de aprendizaje positivos y tener la oportunidad de discutir ciertas creencias puede ser muy útil.

En este Proyecto, partimos de la idea de considerar El Arte en su conjunto como un recurso para contar historias, y esta narración como una forma de participar de experiencias sin tener que vivirlas necesariamente y que, como forma de transmisión de conocimientos y de educación es tan antigua como la historia misma de la humanidad.

El Cine, la séptima de las artes, puede considerarse una de las principales fuentes de generación de narraciones en nuestros días que refleja formas de vida y que, como el resto de las artes, utiliza frecuentemente temas relacionados con la salud, la enfermedad y la medicina entre sus contenidos, diríamos que de forma casi inevitable, mostrándonos realidades y situaciones frente a las cuales, queramos o no, no podemos permanecer ajenos.

Del cine como obra artística podemos decir que engloba al conjunto de las artes, y todos hemos experimentado en alguna ocasión su capacidad para emocionar y conmover el alma², y de aquí su capacidad para formar, potenciar y enriquecer los aprendizajes, y para facilitar la reflexión sobre las realidades que nos rodean y potenciar el desarrollo de nuestras habilidades para la vida $^{3}$ y la construcción de valores ${ }^{4}$.

\section{Objetivos}

\section{Objetivo general}

Contribuir a la Educación para la Salud y a la formación de actitudes y educación de valores saludables en el alumnado mediante la estimulación de la reflexión y el debate grupal utilizando como recurso pedagógico el cine.

\section{Objetivos específicos}

Al finalizar el proyecto, los alumnos serán capaces de:

Conocer valores saludables.

Identificar las actitudes que facilitan el desarrollo de estilos de vida saludable.

Conocer los beneficios/repercusiones de llevar o no un estilo de vida saludable.

Analizar y juzgar los hábitos que siguen en su actual entorno.

Reflexionar y desarrollar estrategias sobre las maneras en las que pueden integrar los conocimientos proporcionados durante el proyecto en su vida diaria.

\section{Metodología}

Al inicio del curso académico 2016-2017, previamente a la puesta en marcha del proyecto se mantuvieron reuniones con los responsables del Centro Docente (I.E.S Gabriel Cisneros de Móstoles) para valorar su idoneidad, alumnos a los que incluir en la actividad y posibilidades de ejecución, siendo aceptado para los tres grupos de alumnos del 4을o de la ESO que han representado la población diana.

En una sesión preliminar con los alumnos y tutores de los respectivos grupos presentamos el proyecto y propuesta de metodología de trabajo en las aulas junto a una selección previa de películas (Tabla 1) cuyos contenidos giran en torno a los tópicos considerados de interés y actualidad referentes a la salud y humanización de la medicina (Tabla 2). A los tutores se les facilitó material de apoyo orientativo para el desarrollo de la actividad5,6.

\section{Contenido de cada sesión}

Previo a la proyección, se facilita a los alumnos en medio impreso, la ficha que contenga los datos técnicos, artísticos y sinopsis, así como el tema o temas en los que se pretende incidir.

El visionado completo de las películas se lleva a cabo 
Tabla 1. Películas inicialmente propuestas.

\begin{tabular}{|c|c|}
\hline Títulos propuestos & Ámbito de salud y valores \\
\hline $\begin{array}{l}\text { Billy Elliot (Quiero bailar) / Billy Eliot (2000) de Stephen Daldry. } \\
111 \text { minutos. }\end{array}$ & $\begin{array}{l}\text { Identidad, creatividad, diferencias } \\
\text { sociales. }\end{array}$ \\
\hline $\begin{array}{l}\text { Quiero ser como Beckham / Bend It Like Beckham (2002) de Gurinder Chadha. } \\
110 \text { minutos. }\end{array}$ & $\begin{array}{l}\text { Afectividad, sexualidad e igualdad de } \\
\text { género. }\end{array}$ \\
\hline $\begin{array}{l}\text { Te doy mis ojos (2003) de Iciar Bollain. } \\
106 \text { minutos. }\end{array}$ & Violencia de género. \\
\hline $\begin{array}{l}\text { Gracias por fumar / Thank You For Smoking (2005) de Jason Reimat. } \\
93 \text { minutos. }\end{array}$ & $\begin{array}{l}\text { El marketing, tabaquismo.(Una película } \\
\text { sobre el tabaco sin fumadores). }\end{array}$ \\
\hline $\begin{array}{l}\text { Juno/ Juno (2007) de Jason Reimat. } \\
92 \text { minutos. }\end{array}$ & Afectividad y sexualidad. \\
\hline $\begin{array}{l}\text { Slumdog Millionaire/ Slumdog Millionaire (2008) de Danny Boyle, Loveleen Tandan. } \\
123 \text { minutos. }\end{array}$ & Glogalización y salud. \\
\hline $\begin{array}{l}\text { Bienvenido al mundo de LOL/ LOL (Laughing Out Loud) (2008) de Lisa Azuelos. } \\
103 \text { minutos. }\end{array}$ & Tabaco, alcohol y otros consumos. \\
\hline $\begin{array}{l}\text { Vivir es fácil con los ojos cerrados (2013) de David Trueba. } \\
105 \text { minutos. }\end{array}$ & Las emociones, despertar sexual. \\
\hline $\begin{array}{l}\text { La familia Bélier/La famille Bélier (2014) de Eric Lartigau. } \\
105 \text { minutos. }\end{array}$ & Discapacidad, amistad, solidaridad. \\
\hline $\begin{array}{l}\text { La profesora de historia/ Les héritiers (2014) de Marie-Castille Mention-Schaar. } \\
100 \text { minutos. }\end{array}$ & Convivencia, resolución de conflictos. \\
\hline $\begin{array}{l}\text { La chica danesa/ The Danish Girl (2015) de Tom Hooper. } \\
120 \text { minutos. }\end{array}$ & Identidad de género, transexualidad. \\
\hline $\begin{array}{l}\text { Yo, él y Raquel / Me \& Earl \& the Dying Girl (2015) de Alfonso Gomez-Rejon. } \\
105 \text { minutos. }\end{array}$ & Adolescencia, amistad, cáncer. \\
\hline $\begin{array}{l}\text { Un monstruo viene a verme (2016) de J.A. Bayona. } \\
100 \text { minutos. }\end{array}$ & $\begin{array}{l}\text { Relaciones materno-filiales, cáncer, acoso } \\
\text { escolar. }\end{array}$ \\
\hline $\begin{array}{l}\text { Por la dignidad de mi hija (TV) / My Daughter's Disgrace (2016) de Monika Mitchell. } \\
105 \text { minutos. }\end{array}$ & Ciberacoso. \\
\hline
\end{tabular}

Tabla 2. Referentes de interés y valores en salud y humanización de la medicina.

Expresión de emociones e identidad.

Importancia de la familia.

Amistad e interculturalidad.

Relaciones sexuales.

Orientación sexual e identidad de genero.

Drogas y otras adicciones.

Violencia de género.

Resolución de conflictos y acoso escolar.

Enfermedad y discapacidad. en dos sesiones de una hora adaptándose a los correspondientes horarios de las tutorías.

Tras el visionado los alumnos trabajan en grupo, sobre el tópico a debate y los aspectos artísticos, técnicos y curiosidades que hayan despertado su interés, recogiendo las conclusiones en el cuadernillo del alumno.

¿Cuál es el mensaje y conceptos clave de la película?

¿En qué medida la película refleja situaciones de la vida real?

¿Qué secuencias han Ilamado especialmente tu atención?

Género cinematográfico, banda sonora, valoración artística y lúdica global. 
Para la evaluación se siguieron las directrices del Servicio Madrileño de Salud en los proyectos de Educación para la Salud de actividades grupales (Grupo: "Sesión de cine en Educación Secundaria". 2017), contemplándose:

Estructura (Idoneidad del local, duración de las sesiones, horario, recursos utilizados).

Proceso (Idoneidad de la actividad y programación, asistencia y participación)

Resultados (Grado de consecución de los objetivos, satisfacción y sugerencias de mejora por parte del profesorado y alumnos.

\section{Resultados y discusión}

El número de alumnos que han participado en la actividad ha sido de 86 , tres tutores de grupo y la orientadora escolar, habiéndose desarrollado un total de siete sesiones.

De forma consensuada los tutores y alumnos, teniendo en cuenta la disponibilidad de tiempo dentro del horario de tutoría, seleccionaron tres títulos, Gracias por fumar / Thank You For Smoking (Figura 1), La profesora de historia / Les héritiers (Figura 2) y La familia Belier / La famille Bélier (Figura 3).

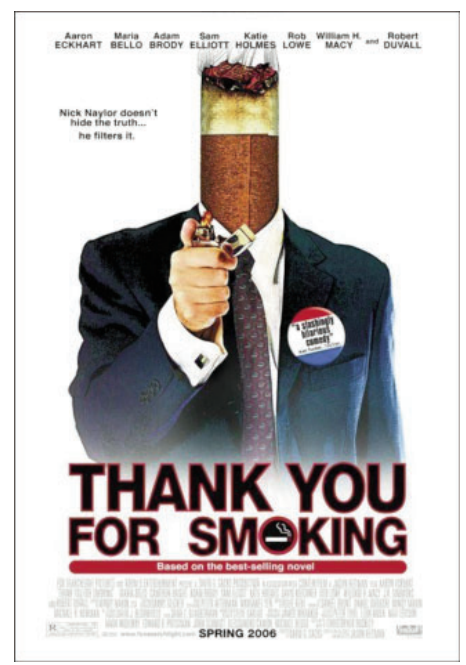

Figura 1. Gracias por fumar / Thank You For Smoking.

Sinopsis: Nick Naylor, jefe de prensa de una gran compañía de tabaco, dedica su vida a defender los derechos de los fumadores contra la cultura neopuritana dominante. Enfrentado a grupos de defensa de la salud y a un oportunista senador, Nick pasa a la ofensiva como relaciones públicas del consumo de cigarrillos, pero al mismo tiempo comienza a pensar en la imagen que está dando a su hijo pequeño Joey. (FilmAffinity).

Enlace:

https://www.filmaffinity.com/es/film803212.html

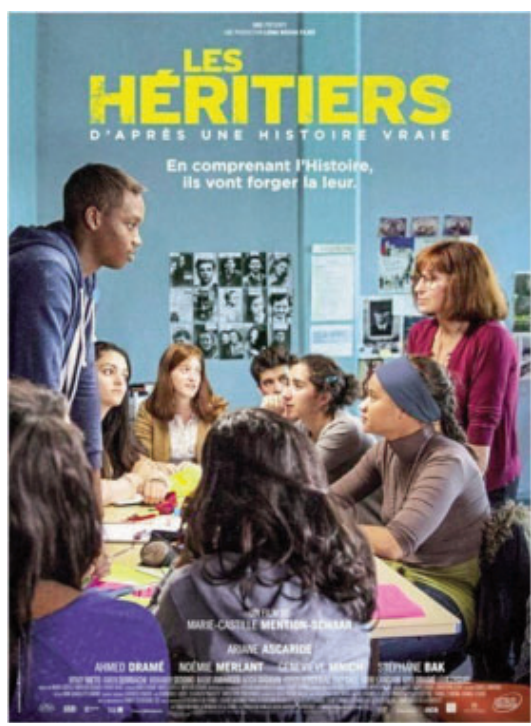

Figura 2. La profesora de historia / Les héritiers.

Sinopsis: Anne Gueguen es una profesora de Historia de instituto que además se preocupa por los problemas de sus alumnos. Este año, como siempre, Anne tiene un grupo difícil. Frustrada por su materialismo y falta de ambición, Anne desafía a sus alumnos a participar en un concurso nacional sobre lo que significa ser adolescente en un campo de concentración nazi. Anne usa toda su energía y creatividad para captar la atención de sus alumnos y motivarlos. A medida que el plazo se acerca, los jóvenes comienzan a abrirse a los demás y a creer en sí mismos. Un proyecto que cambiará sus vidas (FilmAffinity). Enlace:

https://www.filmaffinity.com/es/film819890.html

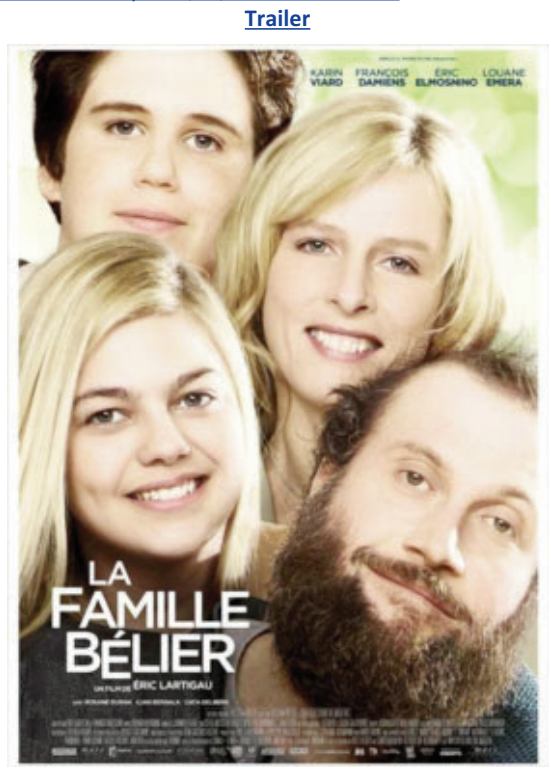

Figura 3. La familia Bélier / La famille Bélier.

Sinopsis: Todos los miembros de la familia Bélier son sordos, excepto Paula, de 16 años. Ella hace de intérprete para sus padres, especialmente en lo que respecta al funcionamiento de la granja familiar. Debido a que a Paula le gusta un chico del instituto, se apunta como él al grupo del coro. Allí descubrirá su talento para el canto... (FilmAffinity).

Enlace:

https://www.filmaffinity.com/es/film406924.html

Trailer 
Según la opinión de los profesores, recogida en la evaluación de la actividad, les permitió trabajar casi la totalidad de las áreas de interés propuestas, especialmente la relevancia de la amistad, expresión de las emociones y de la identidad en la construcción de valores en la adolescencia y juventud, la importancia de la figura de la familia en el desarrollo de su personalidad, el fomento del dialogo para el abordaje y la resolución de conflictos entre iguales y figuras de autoridad, la influencia de los medios de comunicación y del grupo en el tabaquismo y otras adicciones, las relaciones interculturales y la vivencia de la discapacidad como oportunidades de valorar las cualidades de la diferencia.

Entre los aspectos evaluados por los tutores destacar que, la implicación del alumnado y participación en los debates ha sido variable, de media a muy alta dependiendo de cada grupo y en relación con el impacto y nivel de identificación con determinadas situaciones planteadas en las películas.

Ha sido unánime la apreciación de los profesores al considerar la actividad como una buena herramienta para mejorar la calidad de la Educación para la Salud, facilitando el abordaje y discusión de dilemas concretos (identidad, presión de grupo, adicciones, interculturalidad) y en la línea con los objetivos propuestos, recomendando la continuidad de la misma en próximos cursos.

En cuanto a los profesionales del Centro de Salud destacar la acogida positiva por parte de los educadores y alumnado para el desarrollo de proyectos conjuntos y la experiencia de compartir con los usuarios del Centro de Salud espacios "ajenos" que posteriormente hemos encontrado han sido facilitadores de la relación pacienteprofesional de la salud en el entorno sanitario.

\section{Agradecimientos}

A los alumnos y tutores del 4을 curso de la ESO (2016-2017) del IES Gabriel Cisneros de Parque Coímbra (Móstoles) y a Ma Mar Sanz, Orientadora del Centro. Sin la acogida de todos ellos, participación y excelente disposición profesional no hubiera sido posible el desarrollo de éste Proyecto y de otros que de forma conjunta con el Centro de Salud se desarrollan en el ámbito de la Educación para la Salud.

\section{Referencias}

1. Moreta-Velayos F, Moreta-Montero C, Fuente-Hermosín I, Montero-Sánchez N. Sesión de cine en Atención Primaria. Rev Med Cine 2016; 12(1):60-3.

2. Jarné Esparcia IL. Cine y educación: la fuerza de las emociones. En: Cine y habilidades para la vida. Programa Cine y Salud. Nuevas experiencias. Zaragoza: Dirección General de Salud Pública - Gobierno de Aragón. 2007. p. 21-36.

3. Gallego Diéguez J. Habilidades para la vida en la educación para la salud. En: Cine y habilidades para la vida. Programa Cine y Salud. Nuevas experiencias Zaragoza: Dirección General de Salud Pública - Gobierno de Aragón. 2007. p. 11-7. 4. Fernández de Quero J. La construcción de los valores y la necesidad de su educación. Sexpol. 2005; (66):5-6.

5.Gobierno de Aragón. Cine y Salud. Educación para la Salud. Recursos para la Comunidad Educativa [Internet]. 2015.

6.Salud en Curso. Making Of. 2016; (119-120):1-88. 